\title{
UTILIZAÇÃO DE JOGOS PARA PROMOVER O ENGAJAMENTO ESCO- LAR NA APRENDIZAGEM DE CONTEÚDOS TEÓRICOS
}

\author{
Camila Menegali ${ }^{1}$ \\ Luciane Maria Fadel ${ }^{2}$ \\ Maria Collier de Mendonça ${ }^{3}$
}

\begin{abstract}
This paper brings an overview of the research regarding the use of games to promote engagement in the learning of theoretical contents. The use of games has been present in the educational environment for many years, due to the deficiencies noted in traditional teaching regarding student motivation. The student expresses difficulties to realize the practical application of many of the content and constantly considers school activity as something tedious and unpleasant. Through a systematic review, this paper highlights the advantages of using games to support theoretical lecturing, as well as recommendations for a better use of this resource. Keywords: school engagement; learning; games
\end{abstract}

Resumo: Este trabalho analisou a pesquisa realizada na última década a respeito do uso de jogos para promover o engajamento na aprendizagem de conteúdos teóricos. A utilização desse tipo de abordagem está presente no meio educacional há muitos anos, devido às deficiências notadas no ensino tradicional no que se refere à motivação dos alunos. Isto porque é comum o estudante não conseguir perceber a aplicação prática de muitos dos conteúdos, principalmente os de cunho teórico. Estes alunos também consideram a atividade escolar como algo tedioso e pouco agradável. Através de uma revisão sistemática, este artigo aponta algumas das vantagens da utilização de jogos para o ensino de disciplinas teóricas, bem como recomendações para um melhor aproveitamento deste recurso e também as limitações encontradas neste campo de pesquisa.

Palavras-chave: engajamento escolar; aprendizagem; jogos

\section{INTRODUÇÃO}

As desvantagens do ensino tradicional não são uma novidade para os pesquisadores da área da educação. Há anos eles vêm apontando a unilateralidade com que o conteúdo é transmitido (o professor expondo as informações e o estudante no papel de receptor passivo, sem a

\footnotetext{
${ }^{1}$ Programa de Pós-Graduação em Engenharia e Gestão do Conhecimento - Universidade Federal de Santa Catarina (UFSC) Florianópolis - Brasil. Correo electrónico: menegalicamila@ gmail.com

${ }^{2}$ Programa de Pós-Graduação em Engenharia e Gestão do Conhecimento - Universidade Federal de Santa Catarina (UFSC) Florianópolis - Brasil. Correo electrónico: liefadel@ gmail.com

${ }^{3}$ Programa de Pós-Graduação em Engenharia e Gestão do Conhecimento - Universidade Federal de Santa Catarina (UFSC) Florianópolis - Brasil. Correo electrónico: mariacmendonca@ gmail.com
}

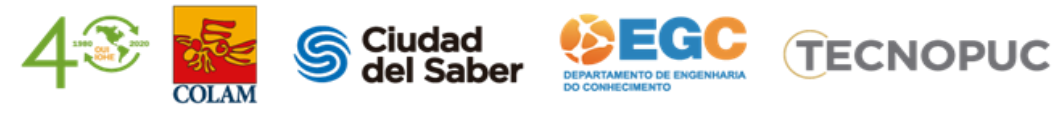


oportunidade de exercer sua criticidade), tornando a aprendizagem mecânica e não capacitando o estudante a exercer seu conhecimento em contextos diferentes daquele em que foi aprendido. (de Castro \& Costa, 2011)

Esse problema acaba se acentuando no ensino teórico, em que os estudantes dificilmente têm uma boa visualização da utilidade desse conhecimento, dificultando a motivação e prejudicando o entendimento. Segundo Barbatsis, Economou, Papamagkana e Loukas (2011), o interesse do estudante pelo conteúdo deve ser evidente e o processo de aquisição de conhecimento deve ser agradável. Pesquisadores como Bodnar e Clark (2014) e Watson e Zaidi (2019) consideram necessário promover o engajamento do estudante no processo de aprendizagem para obter melhores resultados e reter conhecimento.

Uma corrente crescente entre os pesquisadores da educação é o uso de jogos para tornar mais envolventes as atividades escolares, que muitas vezes são consideradas difíceis ou tediosas (Miller, Narayan, Hantsbarger, Cooper \& El-Nasr, 2019). Considerando que os jogos representam um papel cada vez maior na vida dos estudantes (de Sousa \& Rasmussen, 2019) eles podem ajudar a desenvolver uma ponte para uma aprendizagem mais compatível com a realidade.

Ver a educação como uma jornada com o conhecimento como destino seria comparável à experiência de um jogo, exames e práticas são desafios de jogos, resultados de aprendizagem são condições de vitória e, como nos jogos, o objetivo é mudar o jogador/aluno ao longo da jornada. (Williams, 2019, p.351) (tradução nossa)

Diversas são as formas em que os jogos podem ser aplicados na educação. Jogos comerciais como Age of Empires, ou Zoo Tycoon apesar de terem sido desenvolvidos para entretenimento, possibilitam a aquisição de conhecimento (Watson \& Zaidi, 2019) e podem ser utilizados em sala de aula. Existem ainda os jogos que já são desenvolvidos para a aprendizagem, permitindo que os alunos tenham acesso a recursos que são difíceis ou impossíveis de se aplicar no contexto escolar (Rowe, Shores, Mott \& Lester, 2010).

Jogos ou atividades lúdicas, além de auxiliar na educação, também contribuem para construir uma relação positiva entre professor e estudante, mostrando que os dois podem estar inseridos em um contexto de aprendizagem agradável, motivador, prazeroso e rico em possibilidades (de Castro \& Costa, 2011). Além disso, os jogos são uma grande oportunidade de inclusão de alunos com dificuldades de aprendizagem que normalmente se sentem excluídos do

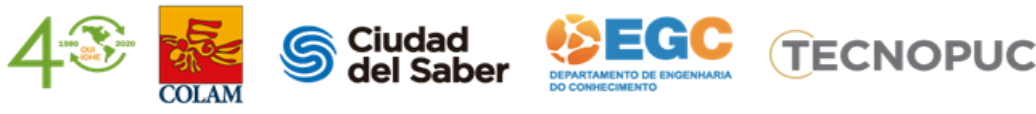


processo tradicional de ensino. Transformar a sala de aula em um ambiente motivador e divertido permite que gradativamente a visão negativa que esses estudantes têm desse espaço vá se modificando, por meio de uma experiência empolgante e desafiadora (Lovato, Christo, Pagliarini, Costa \& Bartholomei-Santos, 2018).

A socialização possibilitada pelos jogos também é um ponto positivo para sua utilização em contexto escolar. Fernandes (2016) e Lovato et al. (2018) demonstraram em seus estudos que o uso de jogos em sala de aula provoca interação entre os estudantes, contribuindo para o desenvolvimento de habilidades de comunicação. O estudo de Mooney e Harkison (2018) apontaram para a oportunidade possibilitada pelo jogo de inserir os estudantes em tarefas comunitárias. Outro ponto levantado pelos autores foi a forma como os jogos facilitam a compreensão de conteúdos teóricos, além de fomentar a conexão da teoria com a prática.

Porém, este é um campo considerado como relativamente incipiente, fazendo com que muitas experiências sejam difíceis ou falhem como fator motivacional (Miller et al., 2019). Por essa razão, uma pesquisa para analisar o estado da arte quanto ao uso de jogos educacionais para auxiliar a aprendizagem de conteúdo teórico mostra-se necessária.

\section{METODOLOGIA}

Para o desenvolvimento desse trabalho, foi realizada uma revisão sistemática nas bases de dados SCOPUS, SCIELO, Web of Science e ACM, pesquisando pelos termos games, engagement ou motivation, education ou learning ou instruction ou teaching e theoretical teaching ou theoretical content utilizando os recursos de truncagem para obter os termos em escrita variada (inclusive recuperando os resultados da palavra "gamification" e "gamificação" ao procurar por gam*) e considerando o período de 2010 até 2020. A pesquisa resultou em um total de 69 artigos, 3 deles sendo duplicados. Após a leitura dos resumos sobraram 24 artigos que se enquadravam no proposto por essa pesquisa. A leitura total dos artigos ainda excluiu mais 3 deles por não terem relevância para o tema, chegando em um total de 21 artigos analisados. 


\section{DISCUSSÃO}

Quanto a distribuição geográfica das publicações, os Estados Unidos lideram a pesquisa na área, com 8 dos 21 artigos analisados tendo sido escritos majoritariamente por autores estadunidenses. Logo em seguida está o Brasil, com 4 publicações selecionadas para a pesquisa, seguido pela Nova Zelândia, com duas publicações. Os países que apresentaram uma publicação selecionada para esta revisão foram: Grécia, Finlândia, Taiwan, Portugal, México, Inglaterra, Noruega, Irlanda e Indonésia. Apesar da pesquisa apresentar publicações de diversos países, não foi possível uma boa representação geográfica no campo em questão. Isto porque, nas bases pesquisadas, não foram encontradas publicações de países africanos e as poucas publicações asiáticas se referem a países do leste da Ásia, não havendo representação do resto do vasto continente.

Analisando os artigos que abordam o uso de jogos para o ensino de conteúdo teórico, notou-se uma tendência de foco em validar ou documentar o uso dos jogos na educação, muitas vezes aplicando o estudo a um jogo específico (ver Tabela 1).

Tabela 1: Artigos que visam validar/documentar o uso de jogos na educação

\begin{tabular}{|l|l|l|}
\hline 2011 & $\begin{array}{l}\text { Contribuições de um jogo didático para o processo de ensino e aprendizagem } \\
\text { de Química no Ensino Fundamental segundo o contexto da Aprendizagem Sig- } \\
\text { nificativa }\end{array}$ & de Castro \& Costa \\
\hline 2011 & Maze: Moodle module for games of exercises & Tiusanen \& Lahtinen \\
\hline 2011 & $\begin{array}{l}\text { Serious games baseados em realidade virtual para educação médica } \\
2012\end{array}$ & $\begin{array}{l}\text { Machado, Moraes, Nu- } \\
\text { nes e Costa }\end{array}$ \\
\hline 2014 & $\begin{array}{l}\text { Exploring the impact game-based learning has on classroom environment and } \\
\text { student engagement within an engineering product design class }\end{array}$ & Tool \\
\hline 2016 & Family Nursing Game: Desenvolvendo um jogo de tabuleiro sobre Família & $\begin{array}{l}\text { Fernes, Gomes e Gonçal- } \\
\text { ves }\end{array}$ \\
\hline
\end{tabular}




\begin{tabular}{|l|l|l|}
\hline 2019 & $\begin{array}{l}\text { Expertise and engagement: re-designing citizen science games with players' } \\
\text { minds in mind }\end{array}$ & Miller et al. \\
\hline 2019 & $\begin{array}{l}\text { Productive disciplinary engagement and videogames: A teacher's educational } \\
\text { design to engage students with ethical theories in citizenship education }\end{array}$ & de Sousa \& Rasmussen \\
\hline 2020 & $\begin{array}{l}\text { The Implementation of Kahoot!: (A Game-Based Student Response System): A } \\
\text { Tool Used in Improving Learning Motivation of Elementary School Students }\end{array}$ & Sartono \\
\hline
\end{tabular}

Fonte: os autores (2020)

Sete, dos vinte e um artigos, tinham o objetivo de propor modelos, frameworks ou estratégias para aprimorar o uso dos jogos na educação (ver Tabela 2).

Tabela 2: Artigos que visam propor modelos, frameworks ou estratégias para o uso de jogos na educação

\begin{tabular}{|l|l|l|}
\hline 2011 & $\begin{array}{l}\text { BD environments with games characteristics for teaching history: the } \\
\text { VRLerna case study }\end{array}$ & Barbatsis et al. \\
\hline 2012 & $\begin{array}{l}\text { Maximizing learning and guiding behavior in free play user generated } \\
\text { content environments }\end{array}$ & $\begin{array}{l}\text { Boyce, Campbell, Pickford, } \\
\text { Culler \& Barnes }\end{array}$ \\
\hline 2013 & $\begin{array}{l}\text { A surrogate competition approach to enhancing game-based learning } \\
\text { Chen \& Chen }\end{array}$ & $\begin{array}{l}\text { Saavedra, Rodríguez, Arteaga \& } \\
\text { De Luna }\end{array}$ \\
\hline 2018 & $\begin{array}{l}\text { In the trail of the genes: a proposal of didactical game for teaching } \\
\text { Genetics }\end{array}$ & Lovato et al \\
\hline 2019 & $\begin{array}{l}\text { Understanding Positive Impact of Game Interactivity in Education } \\
\text { Procling Competencies for Developing Serious Games }\end{array}$ & Watson \& Zaidi \\
\hline for Gameful Learning & $\begin{array}{l}\text { Utilising Game Design to Create Engaging Education: A framework } \\
\text { Williams }\end{array}$ \\
\hline
\end{tabular}

Fonte: os autores (2020)

Alguns artigos (ver Tabela 3) se propuseram a investigar aspectos específicos dos jogos aplicados à aprendizagem, são eles:

Tabela 3: Artigos que investigam aspectos específicos de jogos aplicados à aprendizagem 


\begin{tabular}{|l|l|l|}
\hline 2013 & $\begin{array}{l}\text { Electric agents: fostering sibling joint media engagement through interactive television and } \\
\text { augmented reality }\end{array}$ & $\begin{array}{l}\text { Ballagas } \\
\text { et al. }\end{array}$ \\
\hline 2017 & $\begin{array}{l}\text { Is Difficulty Overrated?: The Effects of Choice, Novelty and Suspense on Intrinsic Motiva- } \\
\text { tion in Educational Games }\end{array}$ & $\begin{array}{l}\text { Lomas et } \\
\text { al. }\end{array}$ \\
\hline 2018 & $\begin{array}{l}\text { Engaging Pictograms! A Methodology for Graphic Design in Enhancing Player Engagement: } \\
\text { As Applied to the Design of a Serious Game for Nepalese Women with Low Literacy }\end{array}$ & $\begin{array}{l}\text { Soriano al. } \\
\text { et an }\end{array}$ \\
\hline
\end{tabular}

Fonte: os autores (2020)

Por fim, dois artigos abordavam aspectos específicos da aprendizagem por meio de jogos, porém focando em considerações referentes a um jogo específico, não propondo a aplicação do estudo a outros jogos (ver Tabela 4).

Tabela 4: Artigos que investigam aspectos específicos de jogos específicos aplicados à aprendizagem

\begin{tabular}{|l|l|l|}
\hline 2010 & $\begin{array}{l}\text { Individual differences in gameplay and learning: a narrative-centered learning per- } \\
\text { spective }\end{array}$ & Rowe et al. \\
\hline 2018 & Assessment for learning in university settings: fun and games & $\begin{array}{l}\text { Mooney \& Harki- } \\
\text { son }\end{array}$ \\
\hline
\end{tabular}

Fonte: os autores (2020)

Vários artigos focaram em oferecer meios de capacitar os educadores para o uso efetivo de jogos em contexto educacional. Bodnar e Clark (2014) levantaram algumas dimensões de interesse da aprendizagem que buscam ser ativadas com o uso dos jogos na educação. Já Saavedra et al. (2016) apresentam o "processo de decomposição baseado em competência" como forma de abranger todo o conteúdo do plano de ensino e permitir maior garantia de aquisição do conhecimento para uma competência específica. Miller et al. (2019) aplicam a "Teoria da Autodeterminação" e a "Teoria da Carga Cognitiva" ao contexto da aprendizagem baseada em jogos. A teoria da autodeterminação identifica os principais fatores intrinsicamente motivadores para os estudantes, a fim de beneficiá-los para promover um sentimento de competência e autorrealização no cumprimento das tarefas propostas. Já a teoria da carga cognitiva defende a 
redução da carga mental para que o foco do estudante seja direcionado à aprendizagem com o jogo.

\subsection{DIFERENTES ASPECTOS REFERENTES A APLICAÇÃO DE JOGOS NA APREN- DIZAGEM}

Diversos fatores influenciam o uso de jogos para promover o engajamento no ensino de conteúdos teóricos. Os artigos incluídos nessa pesquisa abordam não só as vantagens ou aspectos genéricos dessa abordagem como também questões relativas à aprendizagem, competição, narrativa, entre outras especificidades que serão discutidas a seguir.

\subsubsection{Aprendizagem}

As pesquisas sobre jogos aplicados à educação naturalmente levantam questões sobre a natureza do processo de aprendizagem envolvido na atividade. Watson e Zaidi (2019) defendem que o ensino baseado em jogos possui um estilo de aprendizagem ativa. Ao invés de simplesmente receber a informação (como é comum na educação tradicional) os estudantes se envolvem no processo de aprendizagem a partir da mentalidade orientada para objetivos fornecida pelos princípios dos jogos.

Bodnar e Clark (2014) afirmam que as atividades baseadas em jogos expõem os alunos a oportunidades para a tomada de decisões, assim como problemas difíceis em que o conteúdo da sala de aula precisa ser aplicado para a resolução. Essas características oferecem ao estudante um contexto de aprendizagem significativa, que é muito importante principalmente no caso de conteúdos abstratos ou que não estão alinhados à vivência do estudante. O estudo de Castro e Costa (2011) corrobora a afirmação acima. Os autores defendem que as atividades lúdicas auxiliam na apropriação dos conteúdos, permitindo que seja desenvolvido um contexto de aprendizagem significativa.

O fato de estudantes diferentes apresentarem diferenças no conhecimento prévio ou na facilidade com determinadas formas de aprendizagem faz com que seja necessário que essas diferenças sejam levadas em conta na aplicação de jogos para o ensino de conteúdo teórico. Ao

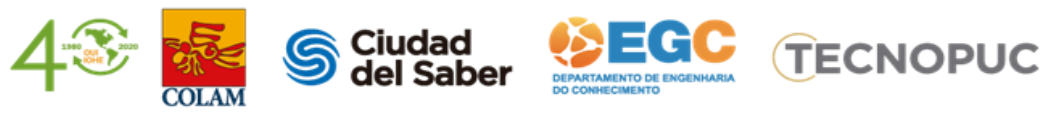


notar que os elementos apresentados no seu experimento não foram suficientes para atingir uma parte considerável dos estudantes envolvidos na pesquisa, Bowe et al. (2010) apontam para a necessidade de apresentar métodos alternativos de apresentação dos conteúdos importantes do jogo educacional, além de incentivos e suporte para direcionar os discentes às atividades importantes, como as atividades de resolução de problemas. Machado et al. (2011) chamam a atenção para a importância do papel do professor em propor, alterar e aprovar as formas de abordagem do conteúdo, a fim de decidir qual é a perspectiva pedagógica mais adequada para cada contexto em que o estudante esteja inserido.

\subsubsection{Colaboração}

O estudo de Ballagas et al. (2013) analisa formas de promover a colaboração entre irmãos durante o processo de aprendizagem, propondo que os jogos educacionais colaborativos podem fazer com o que os irmãos mais velhos ajudem os irmãos mais novos na aquisição do conhecimento. A experiência dos autores feita com crianças submetidas a intervenções com um jogo educacional demonstrou diferentes tipos de colaboração, porém, foram notados alguns comportamentos negativos, sugerindo assim que novas pesquisas sejam feitas para reduzir a incidência desse tipo de situação.

\subsubsection{Competição}

Segundo Chen e Chen (2013) a competição é um fator que traz benefícios à aprendizagem por meio de jogos, como desempenho aprimorado e percepção positiva. Porém, os autores compartilham a preocupação de muitos pesquisadores da área quanto aos possíveis fatores negativos da competição, como vergonha, baixa autoestima e desmotivação. Nesse contexto, desenvolveram o conceito de "competição substituta" em que cada estudante recebe um substituto e a competição com outros estudantes ocorre entre estes substitutos. No modelo proposto pelos autores, os estudantes devem realizar atividades e desafios envolvendo o conhecimento de domínio do jogo educacional. Os pontos adquiridos com a realização dessas atividades servem 
para "nutrir" o substituto, o tornando mais forte para poder competir com os substitutos de outros estudantes.

Na competição substituta, o fator que influencia a vitória ou derrota não é a habilidade no conteúdo em questão, mas seu esforço no processo de aprendizagem. O estudante que vence se sente recompensado, enquanto o estudante perdedor, ao invés de ter sua autoimagem afetada, aprende que o fracasso é causado pela falta de esforço, e não de habilidade, entendendo que precisa se empenhar mais se quiser ganhar a próxima competição (Chen \& Chen, 2013). Os testes realizados pelos autores mostraram que os estudantes submetidos à competição substituta tiveram percepções mais fortes de objetivo, pelo fato de o esforço ser um fator que está sob o controle do aluno, diferente da habilidade.

\subsubsection{Dificuldade}

O papel da dificuldade na aprendizagem baseada em jogos também é discutido na literatura. Lomas et al. (2017) questionam se os benefícios ocasionados pelo desafio são estritamente ligados à dificuldade ou se podem vir de outros fatores. Eles apontam que, ao aumentar a dificuldade, os jogos também apresentam novos elementos de design que atraem a atenção dos estudantes, afirmando que alguns jogos considerados tediosos não faltem em dificuldade, mas em elementos novos e interessantes. $\mathrm{O}$ experimento feito pelos autores também concluiu que o conhecimento do nível de dificuldade da tarefa a ser realizada afeta a interpretação do desempenho do estudante. Tarefas rotuladas como fáceis fazem com que os jogadores tenham menos orgulho de sua realização e mais vergonha ao enfrentar uma derrota.

\subsubsection{Narrativa}

Outra questão importante a ser considerada ao usar jogos em ambientes educacionais é a narrativa. Rowe et al. (2010) afirmam que ambientes de aprendizagem centrados na narrativa promovem atividades cognitivas como suspensão da descrença e envolvimento na história, fatores que permitem melhor contextualização do conteúdo e promovem o engajamento com a

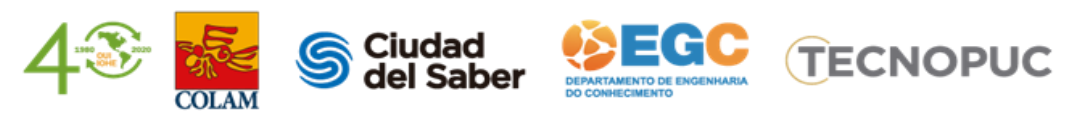


aprendizagem. Machado et al. (2011) atentam para a importância do espaço para interferência do estudante no decorrer da narrativa do jogo ou ambiente de aprendizagem baseado em jogos. As escolhas do usuário podem ser utilizadas para ajustar o nível de dificuldade ou reforçar a aquisição de algum conceito não bem assimilado. Porém, é preciso assegurar que os desafios cubram a totalidade do conteúdo proposto, independente do caminho que o estudante realizar durante a intervenção com o objeto de aprendizagem.

\subsubsection{Elementos visuais e estética}

Segundo Soriano et al. (2018), ao se utilizar de jogos no processo de aprendizagem, os elementos visuais devem ser desenvolvidos tendo em mente tanto seu significado quanto seu impacto no processo cognitivo do estudante. Ao impor decisões ao jogador, é necessária a utilização de imagens que se baseiam no conhecimento do mesmo, pois este precisa às relacionar ao seu significado para manter o fluxo do jogo. Elementos gráficos que devem ser entendidos rapidamente ou passar informações precisas necessitam de simplicidade, enquanto imagens mais complexas são aconselháveis quando se deseja criar um efeito emocional e envolvente.

\subsubsection{Criação de conteúdo personalizado}

Boyce et al. (2012) defendem que a oportunidade do estudante criar seu próprio conteúdo personalizado em jogos educacionais pode ser um fator altamente motivador e também possibilitar a reutilização do sistema. Os autores recomendam que o processo de aprendizagem seja inserido na criação do conteúdo personalizado, a fim de que a aquisição de conhecimento não perca seu apelo para uma atividade não relacionada com a mesma. Porém, alertam para a necessidade do cuidado em não limitar a criatividade dos estudantes.

\subsection{LACUNAS}

Analisando os artigos presentes nessa revisão, foram notadas algumas lacunas no campo de pesquisa. A mais presente foi a dificuldade de generalização dos resultados, pois, em geral,

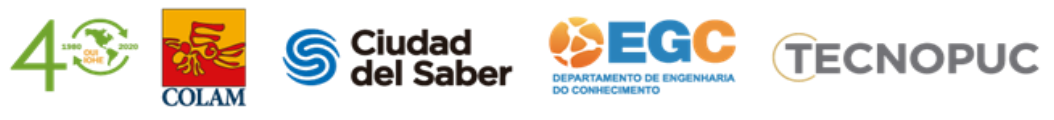


os testes foram feitos em circunstâncias muito específicas. É difícil afirmar que os resultados de um experimento aplicado a apenas uma disciplina, com estudantes de idades semelhantes e com um mesmo repertório cultural possam ser considerados em outros contextos.

Outro fator limitante foi a ausência de aplicação de pré-teste em alguns dos trabalhos experimentais apresentados nesta pesquisa, além da ausência de grupo de controle, o que prejudica a mensuração dos resultados. A falta de precisão dos métodos escolhidos por alguns dos autores presentes nesta revisão também é uma questão preocupante. Apesar do engajamento no ensino teórico ser uma questão subjetiva e de difícil mensuração, a união de métodos qualitativos com quantitativos, o uso de questionários com Escala Likert ${ }^{4}$ ou a análise $\operatorname{dos} \log s^{5}$ dos jogos (no caso de jogos eletrônicos) podem contribuir para uma maior precisão dos resultados. Muitos experimentos, por terem sido aplicados em apenas uma ou poucas sessões, não puderam analisar como a aplicação contínua da abordagem influenciaria no engajamento durante o semestre ou ano letivo. Alguns dos estudos que pretendiam analisar aspectos específicos da utilização de jogos na aprendizagem não isolaram as variáveis corretamente, prejudicando a compreensão dos resultados já que outras variáveis podem ter os influenciado.

\subsection{MÉTODOS DE MENSURAÇÃO DE RESULTADOS}

Pelo fato de muitos artigos encontrados nesta revisão se tratarem de experimentos, uma análise quanto aos métodos de aquisição de dados mostra-se relevante. O método mais utilizado pelos pesquisadores foi o questionário, e a análise do seu uso apresentou questões importantes sobre sua validação. Alguns dos autores optaram por aplicar questionários já prontos, utilizados em outras pesquisas, esta é uma estratégia interessante pois estes já foram validados por outros pesquisadores. Outros autores fizeram seus próprios questionários, nesse caso, o problema da validação foi contornado por alguns ao submetê-los à análise de especialistas. Alguns artigos não apresentaram validação do questionário, o que comprometeu sua confiabilidade. A

\footnotetext{
${ }^{4}$ Escala Likert é um tipo de escala de respostas utilizadas em questionários para medir o quanto o participante concorda ou discorda de uma afirmação.

${ }^{5}$ Os $\log s$ registram informações importantes sobre a utilização de um sistema computacional. Em jogos eletrônicos, eles informam dados sobre o comportamento do jogador, detalhes da partida ou problemas técnicos.
}

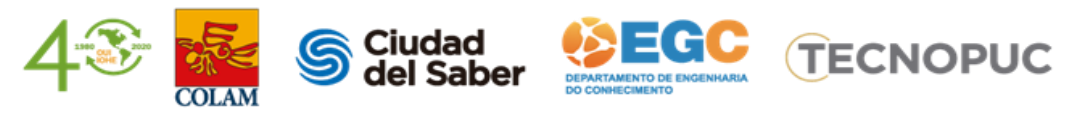


observação direta também esteve muito presente nos experimentos nesta pesquisa. Essa técnica é adequada para colher dados subjetivos e detalhes quanto ao comportamento dos estudantes durante a intervenção.

Apesar da maioria dos dados coletados nos estudos incluídos neste trabalho serem dados qualitativos, o uso de testes de aquisição de conhecimento e a análise de $\log s$ dos jogos (no caso de jogos eletrônicos) se destacaram como métodos de coleta de dados quantitativos. Uma limitação encontrada nos estudos, porém, foi o uso desses métodos isoladamente, sem o auxílio de um método qualitativo, o que tornou os resultados pouco profundos considerando a natureza subjetiva desse campo de pesquisa. Os experimentos que uniram métodos qualitativos e quantitativos de aquisição de dados apresentaram maior profundidade e confiabilidade, mostrando que esta é uma forma mais adequada de se fazer experimentos neste campo de pesquisa.

Outros métodos utilizados nos experimentos em questão foram pesquisas demográficas, entrevistas, taxas de aprovação, pesquisas de satisfação anônimas entre outros.

\section{CONCLUSÃO}

A análise dos artigos presentes nessa revisão revelou que os jogos promovem o engajamento escolar na medida em que fornecem ao estudante maior controle do seu processo de aprendizagem, facilitam a visualização da utilidade e aplicação do conhecimento aprendido, promovem a colaboração, a socialização, o envolvimento, a imersão e a criatividade e facilitam a inclusão de estudantes que apresentam dificuldades com o ensino tradicional. Entre as recomendações apresentadas pelos pesquisadores para a aplicação de jogos na aprendizagem estão o desenvolvimento de narrativa, a análise adequada dos objetivos educacionais para que o resultado da aplicação dos jogos seja compatível com os objetivos pedagógicos, o incentivo à colaboração entre os jogadores e ao desenvolvimento da criatividade e o cuidado com da utilização de competição, dificuldade e elementos gráficos.

Este estudo também revelou lacunas no campo de pesquisa, como a dificuldade de generalização dos resultados e a falta de confiabilidade dos métodos de aquisição de dados encontradas em alguns dos estudos. É importante que os pesquisadores que desejam contribuir

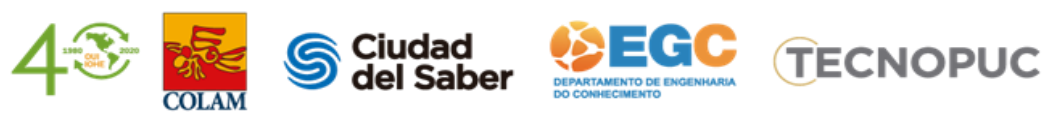


para a área estejam cientes dessas lacunas para tentar preenche-las com seus trabalhos futuros, tornando a literatura mais rica e completa.

\section{AGRADECIMENTOS}

O presente trabalho foi realizado com apoio da Coordenação de Aperfeiçoamento de Pessoal de Nível Superior - Brasil (CAPES) - Código de Financiamento 001.

\section{REFERÊNCIAS}

Ballagas, R., Dugan, T. E., Revelle, G., Mori, K., Sandberg, M., Go, J., Reardon, E., \& Spasojevic, M. (2013, February). Electric agents: fostering sibling joint media engagement through interactive television and augmented reality. In Proceedings of the 2013 conference on Computer supported cooperative work, 225-236.

Barbatsis, K., Economou, D., Papamagkana, I., \& Loukas, D. (outubro de 2011). 3D environments with games characteristics for teaching history: the VRLerna case study. In Proceedings of the 29th ACM International Conference on Design of communication, 5966.

Bodnar, C. A., \& Clark, R. M. (2014, October). Exploring the impact game-based learning has on classroom environment and student engagement within an engineering product design class. In Proceedings of the Second International Conference on Technological Ecosystems for Enhancing Multiculturality, 191-196.

Boyce, A., Campbell, A., Pickford, S., Culler, D., \& Barnes, T. (2012, July). Maximizing learning and guiding behavior in free play user generated content environments. In Proceedings of the 17th ACM annual conference on Innovation and technology in computer science education, 10-15.

Chen, Z. H., \& Chen, S. Y. (2013). A surrogate competition approach to enhancing game-based learning. ACM Transactions on Computer-Human Interaction (TOCHI), 20(6), 1-24. 
de Castro, B. J., \& Costa, P. C. F. (2011). Contribuições de um jogo didático para o processo de ensino e aprendizagem de Química no Ensino Fundamental segundo o contexto da Aprendizagem Significativa. Revista electrónica de investigación en educación en ciencias, 6(2), 25-37.

de Sousa, F., \& Rasmussen, I. (2019). Productive Disciplinary Engagement and Videogames. Nordic Journal of Digital Literacy, 14(03-04), 99-116.

Fernandes, C. S., Martins, M. M., Gomes, B. P., Gomes, J. A., \& Gonçalves, L. H. T. (2016). Family Nursing Game: desenvolvendo um jogo de tabuleiro sobre família. Escola Anna Nery Revista de Enfermagem, 20(1), 33-37.

Lomas, J. D., Koedinger, K., Patel, N., Shodhan, S., Poonwala, N., \& Forlizzi, J. L. (2017, May). Is difficulty overrated? The effects of choice, novelty and suspense on intrinsic motivation in educational games. In Proceedings of the 2017 CHI conference on human factors in computing systems, 1028-1039.

Lovato, F. L., de Christo, T. M., Pagliarini, D. S., Costa, F. D. R., \& Bartholomei-Santos, M. L. (2018). In the trail of the genes: a proposal of didactical game for teaching Genetics. REVISTA DE ENSINO DE BIOQUIMICA, 16(2), 5-30.

Machado, L. D. S., Moraes, R. M. D., Nunes, F. D. L. D. S., \& Costa, R. M. E. M. D. (2011). Serious games baseados em realidade virtual para educação médica. Rev. bras. educ. méd, 254-262.

Miller, J. A., Narayan, U., Hantsbarger, M., Cooper, S., \& El-Nasr, M. S. (2019, August). Expertise and engagement: re-designing citizen science games with players' minds in mind. In Proceedings of the 14th International Conference on the Foundations of Digital Games, 1-11.

Mooney, S., \& Harkison, T. (2018). Assessment for learning in university settings: fun and games. Anatolia, 29(4), 507-517.

Rowe, J. P., Shores, L. R., Mott, B. W., \& Lester, J. C. (2010, June). Individual differences in gameplay and learning: a narrative-centered learning perspective. In Proceedings of the Fifth International Conference on the Foundations of Digital Games, 171-178. 
Saavedra, A. B., Rodríguez, F. J. Á., Arteaga, J. M., \& de Luna, A. C. O. (2016). Process for modeling competencies for developing serious games. REDIE. Revista Electrónica de Investigación Educativa, 18(3), 146-160.

Soriano, D., Arjyal, A., Baral, S., Boscor, A., Mueller, S., Hearn, G. J., ... \& Kostkova, P. (2018, April). Engaging Pictograms! A Methodology for Graphic Design in Enhancing Player Engagement: As Applied to the Design of a Serious Game for Nepalese Women with Low Literacy. In Proceedings of the 2018 International Conference on Digital Health, 30-34.

Sartono, E. K. E. (2020, January). The Implementation of Kahoot! (A Game-Based Student Response System): A Tool Used in Improving Learning Motivation of Elementary School Students. In Proceedings of the 2020 11th International Conference on E-Education, E-Business, E-Management, and E-Learning, 275-278.

Tiusanen, M., \& Lahtinen, R. (2011, November). Maze: Moodle module for games of exercises. In Proceedings of the 11th Koli Calling International Conference on Computing Education Research, 76-82.

Tool, V. T. (2012). "Quem Sou Eu? Jogo dos Vírus": Uma Nova Ferramenta no Ensino da Virologia. Revista Brasileira de Educação Médica, 36(2), 264-268.

Watson, Z., \& Zaidi, S. F. M. (2019, October). Understanding Positive Impact of Game Interactivity in Education. In Proceedings of the 2019 International Conference on Video, Signal and Image Processing, 118-123.

Williams, D. (2019, July). Utilising Game Design to Create Engaging Education: A framework for Gameful Learning. In Proceedings of the 2019 ACM Conference on Innovation and Technology in Computer Science Education, 351-352. 